\title{
Review of the association between periodontitis and chronic obstructive pulmonary disease in smokers
}

\author{
Agathi Spiropoulou ${ }^{1,2}$, Nicholas Zareifopoulos ${ }^{2}$, Aggeliki Bellou ${ }^{2}$, Kostas Spiropoulos ${ }^{1}$, Lazaros Tsalikis ${ }^{1}$ \\ ${ }^{1}$ School of Dentistry, Aristotle University of Thessaloniki; ${ }^{2}$ Department of Pulmonology, School of Medicine, University \\ of Patras, Greece
}

\begin{abstract}
Both periodontitis and chronic obstructive pulmonary disease (COPD) are among the most common diseases associated with smoking. These conditions frequently present alongside comorbidities including diabetes, coronary heart disease, duodenal ulcer, deep vein thrombosis, pulmonary embolism, osteoporosis and muscle atrophy. Chronic inflammation contributes to the pathology of both periodontitis and COPD, and in patients suffering from both conditions treatment of periodontitis may lead to relief from COPD symptoms as well. Smoking contributes to the underlying pathophysiology by causing local inflammation, increasing the production of proinflammatory cytokines and most importantly, by locally increasing the activity of proteolytic enzymes which degrade the extracellular matrix in both periodontal and lung interstitial tissue. The increase in protease activity and extracellular matrix degradation may explain why periodontitis and COPD comorbidity is so common, a finding which also indicates that therapeutic interventions targeting protease activity and the inflammatory response may be beneficial for both conditions.
\end{abstract}

Correspondence: Nicholas Zareifopoulos, Department of Pulmonology, School of Medicine, University of Patras, Rion, Patras 26500, Achaea, Greece.

Tel. +30.2610.312905.

E-mail: nizareifopoulos@gmail.com

Key words: Chronic obstructive pulmonary disease; edentulism; inflammation; metalloproteinase; periodontitis.

Conflict of interest: The authors report no conflict of interest.

Funding: No funding was provided for this work.

Received for publication: 20 December 2018.

Accepted for publication: 25 January 2019.

(C) Copyright A. Spiropoulou et al., 2019

Licensee PAGEPress, Italy

Monaldi Archives for Chest Disease 2019; 89:1018

doi: 10.4081/monaldi.2019.1018

This article is distributed under the terms of the Creative Commons Attribution Noncommercial License (by-nc 4.0) which permits any noncommercial use, distribution, and reproduction in any medium, provided the original author(s) and source are credited.

\section{Introduction}

Periodontal disease is considered to have multiple risk factors. The term "risk factor" refers to the aspect of personal behavior and lifestyle, environmental exposure or an inherited characteristic, which on the basis of epidemiologic evidence is known to be associated with a health-related condition. Risk factors are commonly associated with the pathogenesis of an illness and contribute to the development of the underlying pathophysiological processes either directly or indirectly.

Smoking is a well-established risk factor for periodontitis and bacterial plaque [1]. It is also the most important risk factor for the development of Chronic Obstructive Pulmonary Disease (COPD). It has also been associated with loss of periodontal attachment and bone loss, with individuals with a greater lifetime exposure exhibiting increased risk [2-4]. In cross sectional studies, it is clear that smokers are two to seven times more likely to present periodontitis compared to non-smokers [5-10]. Smoking has also been associated with tooth loss during periodontal maintenance [11,12]. With respect to surgical or non-surgical periodontal therapy, several studies suggest that smoking also compromises the efficacy of treatment and increased the risk for disease recurrence [13].

A recent study [14] evaluated the association between the periodontal status in patients with COPD compared to smokers without COPD, and demonstrated that COPD is an independent risk factor for periodontitis and tooth loss. This study supports also the idea that poor nutritional status (as this confirmed by low serum albumin) in COPD patients might be direct correlated with the reduced chewing ability secondary to periodontitis. Another study conducted in Brazil [9] estimated that smoking cessation programs could result to an approximate reduction of up to $12 \%$ of the number of cases of destructive periodontal disease.

Global trends on tobacco consumption suggest that by 2030, it would be one of the leading causes of death with 8 million people per year dying of smoking-related illnesses. It has been reported that smoking reduces the lifespan of an individual by 7 years [15-17].

Smoking has also been associated with cancer, coronary heart disease, Alzheimer's, cerebrovascular disease and reduced bone mineral density $[18,19]$. The destructive effects of the aforementioned conditions are mediated through toxic combustion and pyrolysis by-products that trigger the inflammatory response [20]. The production of pro-inflammatory cytokines, such as tumor necrosis factor -a (TNF-a), IL-1, IL-6, IL8, is increased, while that of others such as IL-10, is decreased. Additionally, they activate the function of macrophages and dendritic cells. The level of IgE is elevated leading to immune hypersensitivity reactions. It even weakens the innate defenses against pathogens, alters antigen presentation and promotes autoimmunity [20,21]. 
It is widely known that both COPD and periodontitis are characterized from neutrophilic inflammation. As mentioned above, patients with periodontitis plus COPD have higher levels of inflammatory cykokines and exhibit increased C-Reactive Protein (CRP) levels, which is a non-specific marker of inflammation [22,23]. Macrophages secrete also IL-4 and IL-13 which cause airway hyperactivity and mucous production [24].

The relationship between dental health and COPD has not been thoroughly elucidated yet. Several studies suggest that periodontal disease was more common in patients with severe COPD than in others severely ill patients with different chronic conditions $[25,26]$. With this background the current review focuses on the consequences of tobacco smoking on oral health and COPD in adults and the benefits of smoking cessation. It is also intended to clarify the factors underlying increased susceptibility to the harmful effects of smoking and the association between chronic airflow limitation and periodontitis among smokers.

\section{Tobacco smoking as a risk factor for "inflammaging"}

According to the World Health Organization (WHO) "tobacco is one of the greatest emerging disasters in human history." Recently, it was reported that smoking kills more than 5 million people per year worldwide, which is more than HIV/tuberculosis and malaria together [27]. Cigarette smoking is more prevalent among males (46\% in Europe and 56\% in Western Pacific). The proportion of female smokers is less than that of males in middle and high income countries (approximately $50 \%$ of men versus $20 \%$ of women) [26].

Recently, the concept of inflammaging has emerged, which explains the higher basal inflammatory state, characterized by increased circulating cytokines (IL-6, IL1 and TNF-a) in smokers in the absence if an immune challenge [28-31]. Although a number of studies have considered smoking as a true risk factor for periodontitis and COPD, the mechanisms involved are still not clear. A recent in vitro study [32] showed that nicotine in association with lipopolysaccharide (LPS) from periodontopathogenic bacteria increase the levels of IL- 6 as well as IL- 8 protein production by human gingival fibroblasts. Another study found that a similar response could be induced by nicotine alone [33]. Higher levels of TNF-a and IL-8 were observed in the gingival crevicular fluid in smokers compared to non-smokers [34]. It seems that cigarette smoking contains potent inhibitors of gene expression and protein production at least for IL-1b, IL8, IL-2 and TNF-a [35]. These studies although significant, do not provide an accurate description of the underlying mechanism by which smoking affects periodontium. Another preclinical study [36] investigated the profile of a number of pro anti-inflammatory cytokines plus pro and anti-resorptive agents in the gingival tissues of smokers versus non-smokers with moderate to severe chronic periodontitis. It was found that IL-1B, IL-8, IL-10, TNF-a, MMP-8, CRP and osteoprotegerin were lower in smokers, whereas IL- 6 and IFN- $\gamma$ were higher. It was also demonstrated that smoke inhalation enhances periodontal bone destruction in ligature-induced periodontitis [37]. An additional analysis of gingival tissue adjacent to periodontitis sites showed that matrix metalloproteinase (MMP) 2 levels were higher in the exposed versus nonexposed animals. These findings suggest that MMP-2 may be one of the molecules responsible for the increased tissue degradation observed in the periodontal tissue of smokers.

The above serum biomarkers of inflammation especially IL-6, IL-8 C5a, LTB4, MMP1, MMP-3, MMP-7, MMP-9, MMP-12,
MT-IMMP, CXCL10, CXCR2, CRP, TNF-a have been associated also with COPD morbidity [38-40]. Other authors [41] investigated the potential role of these parameters as an association between oral disease and COPD morbidity. Subjects with higher levels of serum IL-6 and CRP had higher event rates. For example, subjects with the lowest levels of IL-6 experienced less common COPD exacerbation $(75 \%)$ than those in higher levels of the abovementioned cytokine $(41.8 \%)$.

\section{Edentulism and COPD related events}

It is known that edentulous (total loss of teeth) individuals who had been diagnosed with COPD, were at greater risk of having a COPD related event (hospitalization and death) than those who had teeth and were characterized as having a healthy periodontal status. The risk for COPD related events was attributable to both edentulism and elevated serum IL-6 [41]. These are a number of potential mechanisms that may underlie this association and additional factors that may account for the findings. Due to the fact that COPD is associated with an abnormal inflammatory response of the lung parenchyma to inhaled pollutants and gases, another hypothesis for the detection of systemic inflammation on these patients was that systemic inflammation in COPD was originating in a form of "spillover" of the pulmonary compartment [42].

Cigarette smoke both directly and indirectly initiates inflammation, but other factors sustain inflammation later in the disease process in the absence of cigarette smoke. Analysis of end stage lung tissue obtained from lung volume reduction surgery surprisingly displayed intense inflammation with a variety of cell types present, including macrophages, T-lymphocytes and eosinophils $[43,44]$. In this study $[43]$ the average time from smoking cessation was nine years. The mechanism by which inflammation is sustained in the absence of cigarette smoking are not known. It may be partly explained by loss of cilia with bacterial colonization and latent viral infection with adenovirus. Also matrix fragments generated by proteinases might lead to a positive feedback loop whereby inflammatory cell proteinases continue to generate extracellular matrix (ECM) fragments, which recruit and activate inflammatory cells with subsequent release of proteinases and further ECM degradation. The reason which edentulism is predictive of COPD-related events is that patients with edentulism have dentures and it is known that biofilm which forms on dentures can house bacteria, yeasts and fungus that result in inflammatory response from oral tissues. Similar to the biofilm on natural teeth, denture biofilm is complex due to the types of organisms it contains, as well as its organized structure. Recently a report [45] was published on the complex nature of the microbial flora contained within the denture biofilm, identifying over 900 individual species of aerobic and anaerobic bacteria, yeasts and amoebae. Others have reported similar complexities for the microbial content of denture plaque. It has also been long recognized that unlike the dental biofilm, the biofilm that forms on denture materials harbors a much larger population of yeasts, including Candida. Candida albicans in particular has been associated with the presence of denture stomatitis. Since periodontal disease does not lead to major tooth loss in a subset of the population that have a strong inflammatory response to infection, it is likely that infections on dentures will lead to a similar inflammatory response. The increased levels of the inflammatory markers that parallel the severity of periodontal disease in association with higher incidence of COPD-related events support a potential systemic origin of the inflammation and 
additionally suggest that edentulism and severe periodontitis are relevant factors in the morbidity of COPD patients [41].

Periodontal disease has been associated with a variety of systemic inflammatory conditions and there is evidence to indicate that components associated with host immune and inflammatory responses as well as systemic exposure to oral pathogens are involved. Other studies $[36,37,41]$ have shown that treating periodontal disease results in reduction of serum inflammatory biomarkers indicating that periodontal disease may enhance systemic inflammation

\section{Pathogenesis of periodontitis}

The mechanisms by which smoking contributes to the pathogenesis of periodontitis are not completely clear. It has been reported that smokers may present a significantly greater plaque index and the average number of bleeding sites in smokers is smaller than in non-smokers [46]. Smokers had a higher prevalence of bacterial species related to periodontal disease compared to nonsmokers, including Porphyromonas gingivalis, Aggregatibacter actinomycetencomitans, Bacteroides firsythus, Prevotella intermedia, Fusobacterium nucleatum [46,47]. Recent studies using real time PCR, have demonstrated a positive relationship between degree of smoking and amount of bacteria/probing depth [48,49].

Several in vitro and in vivo studies $[32,33,50,51]$ have investigated the impact of cigarette smoke constituents, including nicotine and cotinine in the periodontal tissues. Nicotine is just one of the toxic compounds of cigarette smoke. It was demonstrated that the cigarette smoke inhalation enhances periodontal bone destruction in ligature-induced periodontitis [52]. Nicotine adversely affects proliferation, attachment and chemotaxis of periodontal ligament cells and induces pro inflammatory cytokine production by human gingival fibroblasts synergistically with lipopolysaccharide from $E$. coli and $P$. gingivalis. It has also been shown that nicotine increases the production of IL-6 and IL-8 [32]. A microarray analysis demonstrated that peripheral blood mononuclear cells exposed for 5 minutes to tobacco smoke presented an elevated expression of 20 genes previously reported to be associated with periodontal pathogenesis [53].

Higher levels of TNF-a and IL- 8 were observed in the gingival crevicular fluid of smokers compared to nonsmokers [33]. In contrast, pro and anti-inflammatory cytokines have been reported to be lower in association with smoking and its compounds. It seems that cigarette smoke contains potent inhibitors of both gene expression and protein production.

\section{Inflammation due to periodontitis: Localized or systemic?}

As the oral cavity is continuous with the airways, it may serve as a point of entry for lower respiratory tract infections [54], through the following mechanisms:

1. Aspiration, especially during the REM phase of sleep when the only respiratory muscle still working is the diaphragm.

2. Enzymes in saliva that modify the mucous secretions of the airways.

3. Cytokines secreted by neutrophils and permit the bacterial adhesion on the epithelial surface.
Porphyromonas gingivalis, Bacteroides gracilis, Fusobacterium nucleutum and F. necrophorum, Peptostreptococcus and Actonomyces are a variety of oral bacteria that have been cultured from bronchial lavage in patients with respiratory infections [45]. Moreover a comprehensive transcriptional analysis showed that periodontal pathogen $P$. gingivalis adapts to the opportunistic nosocomial Acinetobacter baumannii, thus increasing the abundance of the first [55].

Transient bacteremias are common after dental procedures regardless of periodontal status, occurring frequently after mastication or after personal oral hygiene [56-58]. The incidence and intensity of these bacteremias correlate positively with the extent and severity of periodontitis and are in line with histopathologic observations demonstrating disruption of the epithelial integrity of the periodontal pocket, a sizeable ulcerated surface amounting to up 8 to $20 \mathrm{~cm}^{2}$ and the proximity of highly vascularized tissue to the subgingival biofilm [59]. Oral and periodontal bacteria have been occasionally incriminated as causative for infections at distant organs including the lung, the central nervous system or endovascular prostheses suggesting that they are able to establish themselves at extra-oral locations [60].

\section{Pathogenesis of COPD}

Several lines of evidence contribute to current concepts of the pathogeneses of COPD. Epidemiological studies suggest that environmental exposure to cigarette smoke is critical [61,62]. Alfa-1 antitrypsin deficiency is also an established cause of COPD, and in this population the disease presents at a much earlier age, regardless of smoking status. Genetic risk factors in addition to Alfa-1 antitrypsin deficiency have been described but the genes are yet to be identified $[63,64]$. The overall pathogenetic scheme that is emerging for the pathogenesis is that cigarette smoking and less commonly inhalation of other toxic particulates leads to inflammation, with activation and release of elastase and other matrixdegrading proteinases [64,65].

Cigarette smoke leads to recruitment of several inflammatory and immune cell types. These cells release proteinases causing lung tissue destruction. In the normal lung T-lymphocytes in the blood traverse lung tissue and recirculate in regional lymph nodes before returning to the bloodstream. Monocytes enter the lung, differentiate into tissue macrophages and migrate to the alveolar space. In response to cigarette smoke, neutrophils rapidly accumulate in the lung. Recruitment occurs via smoke stimulation of macrophages and structural cells of the lung, such as epithelial cells, resulting in release of neutrophil chemokins, including IL-8, C5a, LTB4 and other mediators. This results in a significant oxidative burden to the lung due to oxidative substances present in smoke and to generated by the recruited inflammatory cells [66]. Oxidative stress also plays a role in cigarette smoke-induced cell death. Fragments of the extracellular matrix proteins such as laminin and fibronectin are also chemotactic for neutrophils and could play a role characterized by matrix proteolysis. Neutrophils also contain preformed proteinases stored in granules. After activation, granules that contain matrix metalloproteinases (particularly MMP1 and MMP-9) are readily released. Granules that contain serine proteinases (neutrophil elastase) are not readily released, although a portion of granules translocate to the cell surface upon activation, where they are difficult to inhibit [63]. COPD is characterized by a gradual and progressive accumulation of macrophages in the lung. Initially, macrophages accumulation is 
most apparent in respiratory bronchioles [64]. COPD is characterized by a gradual and progressive accumulation of macrophages in the lung. Initially, macrophages accumulation is most apparent in respiratory bronchioles [67]. In addition proteolyzed elastin fragments are chemotactic for macrophages $[68,69]$, which produce a variety of MMPs and thus participate directly in lung destruction.

Both CD4 and CD8 cells are also increased in airway walls and alveoli of patients with COPD. Epithelial cells in smokers with COPD have increased expression of CXCL10, the ligand for Tcell CXCR3 [68]. T-cell products, such as CD40, include MMP expression in several cell types including mononuclear phagocytes [70,71]. Cytotoxic T cells may target epithelial cells and induce cell death, particularly in cells with latent viral infection. Other cells such as dendritic cells, eosinophils and mast cells have also been observed in the lung tissue of patients with COPD, but their role is unclear. Finally, we can postulate that cigarette smoke both directly and indirectly initiates inflammation, but other factors sustain inflammation in the absence of cigarette smoke as mentioned before $[43,72]$.

\section{Matrix metalloproteinases: The missing link?}

The matrix metalloproteinases (MMPs) are a family of 24 enzymes that contain a zinc ion at the active site, have overlapping substrate specificity and are inhibited by TIMP5 [39,73]. MMPs can degrade basal membrane and extracellular matrix components and therefore they have been classically viewed as effectors of extracellular matrix hydrolysis. However, MMPs have also regulatory properties, modulating enzymes, chemokine and cytokine activities among others, as well as releasing bioactive molecules from extracellular matrix store through limited proteolysis. Because collagen I is the main component of the extracellular soft and hard periodontal tissues, MMP5 with collagen-degrading properties such as collagenases and gelatinases (MMP-5, MMP-2 and MMP-9) play a pivotal role in the loss of periodontal support [74-79]. Collagenolytic MMPs have widely been demonstrated in inflamed periodontal tissues and in oral fluids by different analytic methods. MMP-5 can be released and activated during periodontal inflammation by pro-inflammatory cytokines, like TNF-a, IL-1b, reactive oxygen species and proteases derived from subgingival biofilm and the host [80]. Accordingly, higher mRNA expression levels of MMP/TIMP ratios for MMP-1, 2 and 9 as well as RANKL/osteoprotegerin ratio, have been reported in gingival tissue from chronic and aggressive periodontitis compared with healthy gingival tissue [81]. Furthermore, comparison between gingival tissue from aggressive periodontitis and chronic periodontitis showed higher expression of the regulatory cytokines IL-10 and IL-4, and reduced IFN- $\gamma$. Besides the importance of a direct collagenolytic role of these MMP in periodontal tissue breakdown, evidence of a relevant MMP regulatory role in periodontitis is emerging. In vitro studies demonstrate the existence of an activation cascade between MMP-14, MMP-13 and MMP-9 [82-84].

Recently, it has been reported that MMP-10 enhance promatrix MMP-9 activation rate, which was further prevented by administration of MMP-13 specific synthetic inhibitor in gingival tissue from patients with periodontis [85]. Higher MMP-14 levels have also been found in gingival tissue from periodontitis subjects compared to controls [86] and reported soluble forms of MMP-14 in periodontitis gingival crevicular fluid $[87,88]$ along with a positive correlation between active MMP-14 and active MMP-13 [89]. These results suggest that collagenolytic MMP-13, MMP-9 and
MMP-14 can potentiate their activities through proteolytic activation during periodontal disease, generating amplifying loops [90]. Several MMPs degrade elastin and hence are likely to contribute especially to emphysema, including MMP-2, MMP-9, MMP-7 and MMP-12. MMP-12 has been detected in macrophages of smokers with COPD to a greater degree than in healthy smokers. A search for genetic polymorphisms discovered polymorphisms in the MMP-1 promoter region (the less active promoter had worse lung function) and in MMP-12, which together correlated with the rate of lung function decline [40].

Several animal models have supported roles for MMP-5 in COPD. Transgenic mice constitutively overexpressing MMP-1 were found to develop air space enlargement [91]. Mice lacing MMP-12 were resistant to long term cigarette smoke-induced emphysema. Subsequent studies have shown that mice deficient in avB6 develop spontaneous emphysema over time [92]. In the absence of avB6, there is no transforming growth factor-B (TGFb). Because TGF-b normally inhibits MMP-12, avB6 mice have 100-fold excess MMP12 and consequently develop emphysema, while mice that inducibly overexpress IL-13 develop inflammation and expression of MMP-9, MMP-12 and cathepsin-S with consequent emphysema.

\section{Smoking cessation in periodontitis and COPD}

It is not clear how long after smoking discontinuance the patient recovers its normal inflammatory conditions. One study [93] suggested that there is an increase of CD8 T-cells and a decrease of the CD4/CD8 ratio within 6 months after smoking cessation. Another [35] stated that it takes more than 8 weeks for levels of IL-1b, IL-8 and TNF-a to return to their normal values and that normal levels of neutrophil function have not been completely recovered after this period. On the other hand analysis of end stage lung tissue obtained from lung volume reduction surgery surprisingly displayed intense inflammation composed of a variety of cell types, including macrophages, T-cells, neutrophils and eosinophils [43]. The mechanism by which inflammation is sustained in the absence of cigarette smoke is still not known. Matrix fragments generated by proteinases might lead to a positive feedback loop whereby inflammatory cell proteinases continue to generate extracellular matrix fragments, which recruit and activate inflammatory cells with subsequent release of proteinases and further extracellular matrix degradation.

It is clear that multiple inflammatory cell types are present and interact to cause periodontitis and COPD. Rather than focusing in individual cell types, it is the interaction among these cells that is the appropriate target of studies of disease pathogenesis. In addition, structural cells not only are an additional source of proteinases, their viability and ability to repair are critical to the structural integrity of the periodontium and lung $[94,95]$.

\section{Conclusions}

Based on the empirical data from studies published in recent years, it seems that smoking is a potent risk factor for the development of both COPD and periodontitis, two conditions which are frequently comorbid. It seems that there is an increased concentration of some inflammatory mediators and a decreased concentration of others concerning the pathogenetic mechanisms of those 
two diseases. The activation of proteolytic enzymes (induced by the interaction between cigarette smoke components and the immune system) which degrade extracellular matrix components is central to the pathogenesis of both conditions and the underlying pathophysiological processes may be linked. Further studies are required to shed light on the association between COPD and periodontitis, and to establish whether modulation of the immune response (for example by administration of immunosuppressant drugs or interleukin antagonists) may be clinically utilized for the treatment of these conditions.

\section{References}

1. Khan S. Effect of smoking on periodontal health. Dis Mon 2011;57:214-7.

2. Bergstrom J. Cigarette smoking as risk factor in chronic periodontal disease. Community Dent Oral Epidemiol 1989; 17:245-7.

3. Haber J, Wattles J, Crowley M, et al. Evidence for cigarette smoking as a major risk factor for periodontitis. J Periodontol 1993;64:16-23.

4. Haber J. Smoking is a major risk factor for periodontitis. Curr Opin Periodontol 1994;12-8.

5. Albandar JM. Global risk factors and risk indicators for periodontal diseases. Periodontol 2000 2002;29:177-206.

6. Burt B. Position paper: epidemiology of periodontal diseases. J Periodontol 2005;76:1406-19.

7. Luzzi LIT, Greghi SLA, Passanezi E, et al. Evaluation of clinical periodontal conditions in smokers and non-smokers. J Appl Oral Sci 2007; 15:512-7.

8. Oppermann RV, Haas AN, Rosing CK, Susin C. Epidemiology of periodontal diseases in adults from Latin America. Periodontol 2000 2015;67:13-33.

9. Susin C, Oppermann RV, Haugejorden O, Albandar JM. Periodontal attachment loss attributable to cigarette smoking in an urban Brazilian population. J Clin Periodontol 2004;31: 951-8.

10. Tomar SL, Asma S. Smoking-attributable periodontitis in the United States: findings from NHANES III. National Health and Nutrition Examination Survey. J Periodontol 2000;71:743-51.

11. Chambrone L, Chambrone D, Lima LA, Chambrone LA. Predictors of tooth loss during long-term periodontal maintenance: a systematic review of observational studies. J Clin Periodontol 2010;37:675-84.

12. Labriola A, Needleman I, Moles DR. Systematic review of the effect of smoking on nonsurgical periodontal therapy. Periodontol 2000 2005;37:124-37.

13. Wan CP, Leung WK, Wong MCM, et al. Effects of smoking on healing response to non-surgical periodontal therapy: a multilevel modelling analysis. J Clin Periodontol 2009;36:229-39.

14. Terashima T, Chubachi S, Matsuzaki T, et al. The association between dental health and nutritional status in chronic obstructive pulmonary disease. Chron Respir Dis 2017;14:334-41.

15. Bernhard D, Moser C, Backovic A, Wick G. Cigarette smoke an aging accelerator? Exp Gerontol 2007;42:160-5.

16. Burns DM. Cigarette smoking among the elderly: disease consequences and the benefits of cessation. Am J Health Promot 2000;14:357-61.

17. Vellappally S, Fiala Z, Smejkalova J, et al. Smoking related systemic and oral diseases. Acta medica (Hradec Kralove) 2007;50:161-6.
18. Rom O, Avezov K, Aizenbud D, Reznick AZ. Cigarette smoking and inflammation revisited. Respir Physiol Neurobiol 2013;187:5-10.

19. Arnson Y, Shoenfeld Y, Amital H. Effects of tobacco smoke on immunity, inflammation and autoimmunity. J Autoimmun 2010;34:J258-65.

20. Lee J, Taneja V, Vassallo R. Cigarette smoking and inflammation: cellular and molecular mechanisms. J Dent Res 2012;91:142-9.

21. Meraw SJ, Mustapha IZ, Rogers RS 3rd. Cigarette smoking and oral lesions other than cancer. Clin Dermatol 1998;16:625-31.

22. Usher AKH, Stockley RA. The link between chronic periodontitis and COPD: a common role for the neutrophil? BMC Med 2013;11:241.

23. Oztekin G, Baser U, Kucukcoskun M, et al. The association between periodontal disease and chronic obstructive pulmonary disease: a case control study. COPD 2014;11:424-30.

24. Kapellos TS, Bassler K, Aschenbrenner AC, et al. Dysregulated functions of lung macrophage populations in COPD. J Immunol Res 2018;2018:2349045.

25. Gottlieb N. Indian cigarettes gain popularity, but don't let the flavor fool you. J Natl Cancer Inst 1999;91:1806-7.

26. Mendez D, Alshanqeety O, Warner KE. The potential impact of smoking control policies on future global smoking trends. Tob Control 2013;22:46-51.

27. West R. Tobacco smoking: Health impact, prevalence, correlates and interventions. Psychol Health [Internet]. 2017/05/28. 2017;32:1018-36.

28. Jurgensen N, Petersen PE, Ogawa H, Matsumoto $\mathrm{S}$. Translating science into action: periodontal health through public health approaches. Periodontol 2000 2012;60:173-87.

29. Weiskopf D, Weinberger B, Grubeck-Loebenstein B. The aging of the immune system. Transpl Int 2009;22:1041-50.

30. Olivieri F, Rippo MR, Prattichizzo F, et al. Toll like receptor signaling in "inflammaging": microRNA as new players. Immun Ageing 2013;10:11.

31. Wolf J, Weinberger B, Arnold CR, et al. The effect of chronological age on the inflammatory response of human fibroblasts. Exp Gerontol 2012;47:749-53.

32. Wendell KJ, Stein SH. Regulation of cytokine production in human gingival fibroblasts following treatment with nicotine and lipopolysaccharide. J Periodontol 2001;72:1038-44.

33. Giannopoulou C, Roehrich N, Mombelli A. Effect of nicotinetreated epithelial cells on the proliferation and collagen production of gingival fibroblasts. J Clin Periodontol 2001;28: 769-75.

34. Giannopoulou C, Cappuyns I, Mombelli A. Effect of smoking on gingival crevicular fluid cytokine profile during experimental gingivitis. J Clin Periodontol 2003;30:996-1002.

35. Morozumi T, Kubota T, Sugita N, et al.Alterations of gene expression in human neutrophils induced by smoking cessation. J Clin Periodontol 2004;31:1110-6.

36. Cesar Neto JB, de Souza AP, Barbieri D, et al. Matrix metalloproteinase-2 may be involved with increased bone loss associated with experimental periodontitis and smoking: a study in rats. J Periodontol 2004;75:995-1000.

37. Cesar-Neto JB, Duarte PM, de Oliveira MCG, et al. Smoking modulates interleukin-6:interleukin-10 and RANKL:osteoprotegerin ratios in the periodontal tissues. J Periodontal Res 2007;42:184-91.

38. Ohnishi K, Takagi M, Kurokawa Y, et al. Matrix metalloproteinase-mediated extracellular matrix protein degradation in human pulmonary emphysema. Lab Invest 1998;78:1077-87. 
39. Shapiro SD, Kobayashi DK, Ley TJ. Cloning and characterization of a unique elastolytic metalloproteinase produced by human alveolar macrophages. J Biol Chem 1993;268: 23824-9.

40. Joos L, He J-Q, Shepherdson MB, et al. The role of matrix metalloproteinase polymorphisms in the rate of decline in lung function. Hum Mol Genet 2002;11:569-76.

41. Peter KP, Mute BR, Doiphode SS, et al. Association between periodontal disease and chronic obstructive pulmonary disease: a reality or just a dogma? J Periodontol 2013;84:1717-23.

42. Hobbins S, Chapple IL, Sapey E, Stockley RA. Is periodontitis a comorbidity of COPD or can associations be explained by shared risk factors/behaviors? Int J Chron Obstruct Pulmon Dis 2017;12:1339-49.

43. Retamales I, Elliott WM, Meshi B, et al. Amplification of inflammation in emphysema and its association with latent adenoviral infection. Am J Respir Crit Care Med 2001;164:469-73.

44. Hogg JC. Role of latent viral infections in chronic obstructive pulmonary disease and asthma. Am J Respir Crit Care Med. 2001;164:S71-5.

45. Tan L, Tang X, Pan C, et al. Relationship among clinical periodontal, microbiologic parameters and lung function in participants with chronic obstructive pulmonary disease. J Periodontol 2019;90:134-40.

46. Bergstrom J, Persson L, Preber H. Influence of cigarette smoking on vascular reaction during experimental gingivitis. Scand J Dent Res 1988;96:34-9.

47. Zambon JJ, Grossi SG, Machtei EE, et al. Cigarette smoking increases the risk for subgingival infection with periodontal pathogens. J Periodontol 1996;67:1050-4.

48. de Lima FR, Cesar-Neto JB, de Lima DR, et al. Smoking enhances bone loss in anterior teeth in a Brazilian population: a retrospective cross-sectional study. Braz Oral Res 2008;22: 328-33.

49. Brook I. The impact of smoking on oral and nasopharyngeal bacterial flora. J Dent Res 2011;90:704-10.

50. Rezavandi K, Palmer RM, Odell EW, et al. Expression of ICAM-1 and E-selectin in gingival tissues of smokers and nonsmokers with periodontitis. J Oral Pathol Med 2002;31:59-64.

51. Giannopoulou C, Geinoz A, Cimasoni G. Effects of nicotine on periodontal ligament fibroblasts in vitro. J Clin Periodontol 1999;26:49-55.

52. Nociti FHJ, Nogueira-Filho GR, Primo MT, et al. The influence of nicotine on the bone loss rate in ligature-induced periodontitis. A histometric study in rats. J Periodontol 2000;71:1460-4.

53. Ryder MI, Hyun W, Loomer P, Haqq C. Alteration of gene expression profiles of peripheral mononuclear blood cells by tobacco smoke: implications for periodontal diseases. Oral Microbiol Immunol 2004;19:39-49.

54. Muthu J, Muthanandam S, Mahendra J. Mouth the mirror of lungs: where does the connection lie? Front Med 2016;10:405-9.

55. Miller DP, Wang Q, Weinberg A, Lamont RJ. Transcriptome analysis of Porphyromonas gingivalis and Acinetobacter baumannii in polymicrobial communities. Mol Oral Microbiol 2018;33:364-77.

56. Iwai T. Periodontal bacteremia and various vascular diseases. J Periodontal Res 2009;44:689-94.

57. Olsen I. Update on bacteraemia related to dental procedures. Transfus Apher Sci 2008;39:173-8.

58. Lockhart PB, Brennan MT, Sasser HC, et al. Bacteremia associated with toothbrushing and dental extraction. Circulation 2008;117:3118-25.

59. Hujoel PP, Drangsholt M, Spiekerman C, DeRouen TA.
Periodontal disease and coronary heart disease risk. JAMA 2000;284:1406-10.

60. Nanci A, Bosshardt DD. Structure of periodontal tissues in health and disease. Periodontol 2000 2006;40:11-28.

61. Elliott WM, Hayashi S, Hogg JC. Immunodetection of adenoviral E1A proteins in human lung tissue. Am J Respir Cell Mol Biol 1995;12:642-8.

62. Matsuse T, Hayashi S, Kuwano K, Latent adenoviral infection in the pathogenesis of chronic airways obstruction. Am Rev Respir Dis 1992;146:177-84.

63. Sandford AJ, Joos L, Pare PD. Genetic risk factors for chronic obstructive pulmonary disease. Curr Opin Pulm Med 2002;8:87-94.

64. Silverman EK, Chapman HA, Drazen JM, et al. Genetic epidemiology of severe, early-onset chronic obstructive pulmonary disease. Risk to relatives for airflow obstruction and chronic bronchitis. Am J Respir Crit Care Med 1998;157:1770-8.

65. Luisetti M, Pignatti PF. The search for susceptibility genes of COPD. Monaldi Arch Chest Dis 1995;50:28-32.

66. Rennard S, Decramer M, Calverley PMA, et al. Impact of COPD in North America and Europe in 2000: subjects' perspective of Confronting COPD International Survey. Eur Respir J 2002;20:799-805.

67. Niewoehner DE, Kleinerman J. Morphologic basis of pulmonary resistance in the human lung and effects of aging. J Appl Physiol 1974;36:412-8.

68. Saetta M, Mariani M, Panina-Bordignon P, et al. Increased expression of the chemokine receptor CXCR3 and its ligand CXCL10 in peripheral airways of smokers with chronic obstructive pulmonary disease. Am J Respir Crit Care Med 2002;165:1404-9.

69. Saetta M, Baraldo S, Corbino L, et al. CD8+ve cells in the lungs of smokers with chronic obstructive pulmonary disease. Am J Respir Crit Care Med 1999;160:711-7.

70. Malik N, Greenfield BW, Wahl AF, Kiener PA. Activation of human monocytes through $\mathrm{CD} 40$ induces matrix metalloproteinases. J Immunol 1996;156:3952-60.

71. Wu L, Fan J, Matsumoto SI, Watanabe T. Induction and regulation of matrix metalloproteinase- 12 by cytokines and CD 40 signaling in monocyte/macrophages. Biochem Biophys Res Commun 2000;269:808-15.

72. Gold DR, Wang X, Wypij D, et al. Effects of cigarette smoking on lung function in adolescent boys and girls. N Engl J Med 1996;335:931-7.

73. Parks WC, Shapiro SD. Matrix metalloproteinases in lung biology. Respir Res 2001;2:10-9.

74. Overall CM. Molecular determinants of metalloproteinase substrate specificity: matrix metalloproteinase substrate binding domains, modules, and exosites. Mol Biotechnol 2002;22:51-86.

75. Sbardella D, Fasciglione GF, Gioia M, et al. Human matrix metalloproteinases: an ubiquitarian class of enzymes involved in several pathological processes. Mol Aspects Med 2012;33:119-208.

76. Sorsa T, Hernandez M, Leppilahti J, et al. Detection of gingival crevicular fluid MMP-8 levels with different laboratory and chair-side methods. Oral Dis 2010;16:39-45.

77. Sorsa T, Tjaderhane L, Konttinen YT, et al. Matrix metalloproteinases: contribution to pathogenesis, diagnosis and treatment of periodontal inflammation. Ann Med 2006;38:306-21.

78. Sorsa T, Tjaderhane L, Salo T. Matrix metalloproteinases (MMPs) in oral diseases. Oral Dis 2004;10:311-8.

79. de Souza AP, Trevilatto PC, Scarel-Caminaga RM, et al. MMP-1 promoter polymorphism: association with chronic 
periodontitis severity in a Brazilian population. J Clin Periodontol 2003;30:154-8.

80. Kowolik MJ, Grant M. Myeloperoxidase activity in human gingival crevicular neutrophils. Arch Oral Biol 1983;28:293-5.

81. Garlet GP, Martins WJ, Fonseca BAL, et al. Matrix metalloproteinases, their physiological inhibitors and osteoclast factors are differentially regulated by the cytokine profile in human periodontal disease. J Clin Periodontol 2004;31:671-9.

82. Folgueras AR, Pendas AM, Sanchez LM, Lopez-Otin C. Matrix metalloproteinases in cancer: from new functions to improved inhibition strategies. Int J Dev Biol 2004;48:411-24.

83. Knauper V, Smith B, Lopez-Otin C, Murphy G. Activation of progelatinase B (proMMP-9) by active collagenase-3 (MMP13). Eur J Biochem 1997;248:369-73.

84. Knauper V, Will H, Lopez-Otin C, et al. Cellular mechanisms for human procollagenase-3 (MMP-13) activation. Evidence that MT1-MMP (MMP-14) and gelatinase a (MMP-2) are able to generate active enzyme. J Biol Chem 1996;271:17124-31.

85. Hamlet SM, Ganashan N, Cullinan MP, et al. A 5-year longitudinal study of Tannerella forsythia prtH genotype: association with loss of attachment. J Periodontol 2008;79:144-9.

86. Oyarzun A, Arancibia R, Hidalgo R, et al. Involvement of MT1-MMP and TIMP-2 in human periodontal disease. Oral Dis 2010;16:388-95.

87. Hernandez M, Gamonal J, Tervahartiala T, et al. Associations between matrix metalloproteinase- 8 and -14 and myeloperoxidase in gingival crevicular fluid from subjects with progressive chronic periodontitis: a longitudinal study. J Periodontol. 2010; 81:1644-52.
88. Tervahartiala T, Pirila E, Ceponis A, et al. The in vivo expression of the collagenolytic matrix metalloproteinases (MMP-2, $-8,-13$, and -14) and matrilysin (MMP-7) in adult and localized juvenile periodontitis. J Dent Res.2000;79:1969-77.

89. Hernandez M, Martinez B, Tejerina JM, et al. MMP-13 and TIMP-1 determinations in progressive chronic periodontitis. J Clin Periodontol 2007;34:729-35.

90. Hernandez M, Gamonal J, Salo T, et al. Reduced expression of lipopolysaccharide-induced CXC chemokine in Porphyromonas gingivalis-induced experimental periodontitis in matrix metalloproteinase-8 null mice. J Periodontal Res 2011;46:58-66.

91. D’Armiento J, Dalal SS, Okada Y, et al. Collagenase expression in the lungs of transgenic mice causes pulmonary emphysema. Cell 1992;71:955-61.

92. Morris DG, Huang X, Kaminski N, et al. Loss of integrin alpha(v)beta6-mediated TGF-beta activation causes Mmp12dependent emphysema. Nature 2003;422:169-73.

93. Bouloukaki I, Tsoumakidou M, Vardavas CI, et al. Maintained smoking cessation for 6 months equilibrates the percentage of sputum CD8+ lymphocyte cells with that of nonsmokers. Mediators Inflamm 2009;2009:812102.

94. Gross P, Babyak MA, Tolker E, Kaschak M. Enzymatically produced pulmonary emphysema: a preliminary report. J Occup Med.1964;6:481-4.

95. Preshaw PM, Heasman L, Stacey F, et al. The effect of quitting smoking on chronic periodontitis. J Clin Periodontol 2005;32: 869-79. 